\title{
INFLUENCIA DE LA EDAD DE LIXIVIADOS SOBRE SU COMPOSICIÓN FÍSICO-QUÍMICA Y SU POTENCIAL DE TOXICIDAD
}

\section{INFLUENCE OF LEACHATES AGE ON ITS PHYSICO- CHEMICAL COMPOSITION AND TOXICITY POTENTIAL}

\begin{abstract}
Patricia Torres-Lozada ${ }^{1}$, Luz Edith Barba-Hoํㄹ ${ }^{2}$ César Ojeda ${ }^{3}$, Jonier Martínez ${ }^{4}$, Yulieth Castaño ${ }^{5}$
${ }^{1}$ Ing. Sanitaria, M.Sc., Ph.D. Prof. Titular, Facultad de Ingeniería, Universidad del Valle, Cali, Colombia, e-mail: patricia. torres@correounivalle.edu.co; ${ }^{2}$ Química M.Sc. Prof. Titular, Facultad de Ingeniería, Universidad del Valle, Cali, Colombia, e-mail: luz.barba@correounivalle.edu.co; ${ }^{3}$ Estadístico, M.Sc. Prof. Auxiliar, Facultad Ingeniería, Universidad del Valle, Cali, Colombia, e-mail: cesar.ojeda@correounivalle.edu.co; ${ }^{4}$ Est. Estadística, Universidad del Valle, Cali, Colombia, e-mail: jonier. martinez@correounivalle.edu.co; ${ }^{5}$ Est. Estadística, Universidad del Valle, Cali, Colombia, e-mail: yulieth.castano@correounivalle.edu.co. Dirección correspondencia: Carrera 100 No. 13-00 Edif.336. Cali, Valle del Cauca-Colombia. e-mail: patricia. torres@correounivalle.edu.co.
\end{abstract}

Rev. U.D.C.A Act. \& DIv. Cient. 17(1): 245-255, Enero-Junio 2014

\section{RESUMEN}

Los lixiviados son subproductos de la operación normal de rellenos sanitarios, que pueden contener una amplia diversidad de contaminantes; las prácticas tradicionales de manejo de los rellenos sanitarios se basan, principalmente, en la caracterización físico-química, debido a su relación con la toxicidad. En el presente estudio, se analizó la influencia de la edad de los lixiviados de celdas dedos rellenos sanitarios y un vertedero sobre las características físico-químicas y su potencial toxicidad. En general, la composición de los lixiviados depende de la edad de la celda en la que se originan, encontrándose mayores concentraciones en los lixiviados más jóvenes, en los cuales, la mayoría de mediciones están asociadas con las variables indicadoras de materia orgánica y sólidos; en los lixiviados de mayor edad, las concentraciones medidas y la relación entre las variables disminuyen, siendo necesario caracterizar un mayor número de variables.

Palabras clave: Caracterización de lixiviados, correlación de parámetros, relleno sanitario municipal, toxicidad.

\section{SUMMARY}

Leachates are byproducts of the normal operation of landfills that may contain a huge diversity of contaminants; traditional landfills management practices are mainly based on physicochemical characterization due to their relationship with toxicity. The aim of this study was to analyze the influence of age leachates of cells from two different landfills and one weir on physicochemical characteristics and their toxicity potential. In general, we observed that the composition of the leachates depends of the age of the cells in which they're originated, finding greater concentrations in younger leachates in which the majority of measures are associated with organic matter and solid variables, in the older leachates, the measured concentrations and the relationship between the variables decreases, being necessary to characterize a greater number of variables.

Key words: Leachate characteristics, statistical correlation of physicochemical parameters, municipal landfill, toxicity.

\section{INTRODUCCIÓN}

La cantidad de residuos generados por la sociedad está ligada estrechamente con su nivel económico; la mayoría de éstos son el resultado de los productos usados que, luego de cumplir la función para la que fueron adquiridos, se convierten en desechos denominados residuos sólidos urbanos - RSU (Shekdar, 2009), cuya eliminación adecuada es una necesidad y hace parte integral del entorno urbano y la planificación de la infraestructura, para garantizar un ambiente seguro y saludable, considerando la promoción del crecimiento económico sostenible (Karak et al. 2013).

La disposición final es un componente importante de la gestión integral de los residuos sólidos - GIRS, debido a que aquellos RSU que no se reutilizan, requieren un lugar dónde depositarlos, de manera definitiva. Los rellenos sanitarios son y seguirán siendo la alternativa de disposición final de RSU más utilizada, tanto en los países en desarrollo como 
en los industrializados (Bartone \& Bernstein, 1993; Themelis, 2003; Hoornweg \& Bhada-Tata, 2012). En Colombia, se mantiene esta tendencia, ya que aunque predominan los botaderos a cielo abierto $(40,9 \%)$ y los rellenos sanitarios (42,4\%), la cantidad de RSU dispuestos en cada uno de ellos es del orden del 7 y 90\%, respectivamente (SSPD, 2010).

Cuando los RSU son dispuestos en rellenos sanitarios ocurren una serie de reacciones biológicas y químicas, que favorecen su degradación, siendo generalmente aceptado que en rellenos sanitarios de 30 años o menos de operación, ocurren, al menos, cuatro fases de descomposición: aerobia inicial, ácida anaerobia, metanogénica inicial y metanogénica estable; sin embargo, factores en el relleno sanitario, como la composición de los RSU, condiciones climáticas, como la precipitación e infiltración, la forma de operación, como es el caso de la recirculación de los lixiviados y la implementación de procesos de aireación, pueden tener un efecto sobre las tasas y el tiempo de degradación de los RSU (Kjeldsen et al. 2002).

La mayoría de los rellenos sanitarios reciben una mezcla de residuos municipales, comerciales e industriales, pero excluyen cantidades significativas de residuos altamente tóxicos o peligrosos (Christensen et al. 1994). Generalmente, el impacto ambiental de los rellenos sanitarios está asociado, principalmente, con la emisión de Gases de Efecto Invernadero - $\mathrm{GEI}\left(\mathrm{CO}_{2} \mathrm{yCH}_{4}\right)$ y los efluentes líquidos o lixiviados producidos, como consecuencia de la percolación de las aguas lluvias, a través de la masa de residuos, las reacciones químicas y biológicas ocurridas en las celdas que contienen los residuos y su contenido de agua (Boumechhour et al. 2013).

Los lixiviados son fuente de sustancias químicas y biológicas altamente tóxicas, que pueden generar perjuicios en la salud humana y el ambiente (Raco et al. 2013); el mayor impacto ambiental está asociado a la contaminación de fuentes de agua superficial y subterránea (Pablos et al. 2011).

Generalmente, los lixiviados se componen de una amplia gama de contaminantes orgánicos e inorgánicos, que pueden ser clasificados en cuatro grupos (Kjeldsen et al. 2002; Wiszniowski et al. 2006; Aziz et al. 2010): i) materia orgánica disuelta (DQO, COT, AGV, ácidos húmicos y fúlvicos); ii) macrocomponentes inorgánicos ( $\mathrm{Ca}, \mathrm{Mg}, \mathrm{Na}, \mathrm{K} \mathrm{NH}_{4}{ }^{+}, \mathrm{Fe}, \mathrm{Mn}$; $\mathrm{Cl}$, $\mathrm{SO}_{4}, \mathrm{HCO}_{3}{ }^{-}$); iii) metales pesados ( $\left.\mathrm{Cd}, \mathrm{Cr}, \mathrm{Cu}, \mathrm{Pb}, \mathrm{Ni}, \mathrm{Zn}\right) \mathrm{y}$, iv) Compuestos xenobióticos (hidrocarburos aromáticos, fenoles, alifáticos clorados, pesticidas, plastificantes). Otros compuestos pueden estar presentes en los lixiviados (boratos, sulfuros, arsenato, selenato, $\mathrm{Ba}, \mathrm{Li}, \mathrm{Hg}, \mathrm{Co}$ ), pero, normalmente, en concentraciones muy bajas (Ramírez - Sosa et al. 2013).

En un mismo relleno sanitario es común encontrar áreas o celdas de diferentes edades, lo que genera lixiviados de di- ferente composición (Ragle et al. 1995; Pablos et al. 2011). Adicionalmente, la composición de los lixiviados varía entre diferentes rellenos sanitarios, dependiendo, principalmente, de factores, como la composición y la etapa de degradación de los RSU y la tecnología o tipología de relleno (Kjeldsen et al. 2002). De acuerdo con diversos autores (Kang et al. 2002; Kulikowska \& Klimiuk, 2008; Renou et al. 2008; Shouliang et al. 2008), los lixiviados se pueden clasificar en tres grupos, de acuerdo con el tiempo de operación del relleno sanitario y, particularmente, de la celda del relleno, de la cual, provengan (jóvenes $<5$ años; intermedios 5-10 años y maduros $>10$ años de operación).

En general, el grado de biodegradabilidad de los lixiviados es inversamente proporcional a su edad, siendo más biodegradables los jóvenes y menos los maduros (Fátima et al. 2012; Ramírez - Sosa et al. 2013), por esta razón, la determinación de la cantidad y la composición de los lixiviados son un paso esencial en el desarrollo de sus sistemas de gestión (AbuQdais et al. 1997; El-Fadel et al. 2002), incluida la identificación y la selección de estrategias de tratamiento adecuadas (Shouliang et al. 2008). En el presente estudio, se analizó la influencia del tiempo de operación de celdas de dos rellenos sanitarios y un vertedero del Valle del Cauca sobre las características físico-químicas de los lixiviados generados y la correlación entre las variables.

\section{MATERIALES Y MÉTODOS}

Fueron analizadas 20 muestras de lixiviados, procedentes de celdas de dos rellenos sanitarios activos (una con una edad o tiempo de operación de 5 años y otra de 15 años) y un vertedero clausurado hace cinco años y que operó durante 51 años, todos ellos, ubicados en el departamento del Valle del Cauca - Colombia. Las muestras fueron tomadas en diferentes periodos de 2012.

Las muestras fueron preservadas a $4^{\circ} \mathrm{C}$ y fueron caracterizadas mediante la determinación de: $\mathrm{pH}$, conductividad (CE); sales: alcalinidad (AT), dureza (DT), cloruros $\left(\mathrm{Cl}^{-}\right)$; materia orgánica: ácidos grasos volátiles (AGV), demanda química y bioquímica de oxígeno (DQO y DBO5), carbono orgánico total (COT); sólidos: sólidos totales y disueltos (ST y SDT) y formas de nitrógeno: nitrógeno total y amoniacal. Adicionalmente, se hicieron mediciones de sustancias tóxicas, como metales pesados, que incluyeron hierro, manganeso, níquel, aluminio cadmio, zinc y plomo; benceno, tolueno, etilbenceno y xileno (BTEX), así como hidrocarburos aromáticos policíclicos (HAP's), siguiendo las metodologías de APHA (2005).

Teniendo en cuenta que la toxicidad del nitrógeno amoniacal es debida a la forma no ionizada (amoniaco libre o $\mathrm{NH}_{3}$ ), para su cálculo, se utilizó la Ecuación 1 (Anthonisen et al. 
1976), que relaciona el $\mathrm{pH}$ de la muestra, la temperatura ambiente y la concentración de nitrógeno amoniacal.

$$
N_{3}(m g / L)=\frac{(17 / 14) *\left(N \text { amoniacal } * 10^{p H}\right)}{\left(\frac{K_{b}}{K_{w}}+10^{p H}\right)} \quad \text { Ec. } 1
$$

Donde, $K_{b} / K_{w}$ es la relación entre las constantes de disociación del amoniaco y el agua con respecto de la temperatura $\left({ }^{\circ} \mathrm{C}\right)$ del medio y equivale a $\mathrm{e}^{\left(6,344 / 273+{ }^{\circ} \mathrm{C}\right)}$.

Inicialmente, los registros fueron analizados estadísticamente, mediante el uso de técnicas descriptivas univariadas, como indicadores de tendencia central, variabilidad, posición, forma y representaciones gráficas. Posteriormente, se utilizó una técnica descriptiva, que permite conocer el grado de asociación lineal entre las variables involucradas y la intensidad de tal asociación, conocida como análisis de correlaciones, considerando, como una fuerte relación lineal entre dos variables, un coeficiente de correlación mayor de 0,85 (Johnson \& Wichern, 2007).

Posterior al análisis descriptivo, se realizó un análisis multivariado, denominado análisis de componentes principales
(ACP), que explica la estructura de correlación del conjunto de variables fisicoquímicas, a través de pocas combinaciones lineales de éstas con el objetivo de reducir dimensionalidad y poder interpretarlas de manera más sencilla.

Los resultados obtenidos fueron procesados a través del paquete estadístico R (R Core Team, 2012), el cual, es de uso libre y es distribuido bajo los términos de la Licencia Pública General (GNU). Para el desarrollo del ACP, se usaron las librerías ade4 (Dray \& Dufour, 2007; Chessel et al. 2004; Dray et al. 2007) y ade4TkGUI (Thioulouse \& Dray, 2009).

\section{RESULTADOS Y DISCUSIÓN}

Análisis Descriptivo: En la tabla 1, se presentan los rangos (valores máximo y mínimo) de las variables fisicoquímicas analizadas a los tres tipos de lixiviados y en las figuras 1 y 2 , se muestra la representación gráfica en función de las medias y su dispersión; en la primera, se presentan las variables DQO, DBO, COT, AGV's, Cloruros y ST, mientras que en la segunda, se encuentran los STD, Conductividad, Alcalinidad, Nitrógeno amoniacal, Nitrógeno total y dureza.

Tabla 1. Resumen de variables fisicoquímicas en los lixiviados estudiados.

\begin{tabular}{|l|c|c|c|c|c|c|c|}
\hline \multirow{2}{*}{ Parámetros } & \multirow{2}{*}{ Unidades } & \multicolumn{2}{|c|}{ LJ } & \multicolumn{2}{c|}{ LI } & \multicolumn{2}{c|}{ LM } \\
\cline { 3 - 8 } & & Max & Min & Max & Min & Max & Min \\
\hline pH & Unidades & 8,26 & 7,77 & 8,5 & 7,6 & 9,58 & 8,18 \\
\hline AGE & $\mathrm{mS} / \mathrm{cm}$ & 36,7 & 27,1 & 23,5 & 16,2 & 20,6 & 11,6 \\
\hline AT & $\mathrm{meq} / \mathrm{L}$ & 295 & 70 & 100 & 50 & 62,5 & 45 \\
\hline DT & $\mathrm{mg} \mathrm{CaCO}_{3} / \mathrm{L}$ & 36300 & 12400 & 10746 & 7344 & 8694 & 1689 \\
\hline DBO 5 & $\mathrm{mg} \mathrm{CaCO} / \mathrm{L}$ & 4324 & 1251 & 1863 & 866 & 2700 & 400 \\
\hline DQO & $\mathrm{mg} \mathrm{O}_{2} / \mathrm{L}$ & 13391 & 1171 & 1594 & 496 & 165 & 78 \\
\hline COT & $\mathrm{mg} \mathrm{O}_{2} / \mathrm{L}$ & 25455 & 9181 & 6638 & 3673 & 2197 & 1105 \\
\hline ST & $\mathrm{mg} \mathrm{COT/L}$ & 7840 & 3531 & 3025 & 1240 & 999 & 415 \\
\hline SDT & $\mathrm{mg} / \mathrm{L}$ & 33796 & 17673 & 17950 & 10596 & 9345 & 5472 \\
\hline NTK & $\mathrm{mg} / \mathrm{L}$ & 33703 & 17041 & 17775 & 10473 & 8877 & 5382 \\
\hline NH 3 Libre & $\mathrm{mg} \mathrm{N-NTK/L}$ & 2492 & 2184 & 2072 & 1204 & 1095 & 9,2 \\
\hline N Amoniacal & $\mathrm{mg} \mathrm{N-NH} / \mathrm{L}$ & 2184 & 1050 & 1848 & 1008 & 956 & 9,2 \\
\hline Cl & $\mathrm{mg} \mathrm{Cl} / \mathrm{L}$ & 4200 & 2121 & 3099 & 1398 & 2420 & 800 \\
\hline
\end{tabular}

LJ: Lixiviado joven $<5$ años

LI: Lixiviado intermedio 5-10 años

LM: Lixiviado Maduro > 10 años 

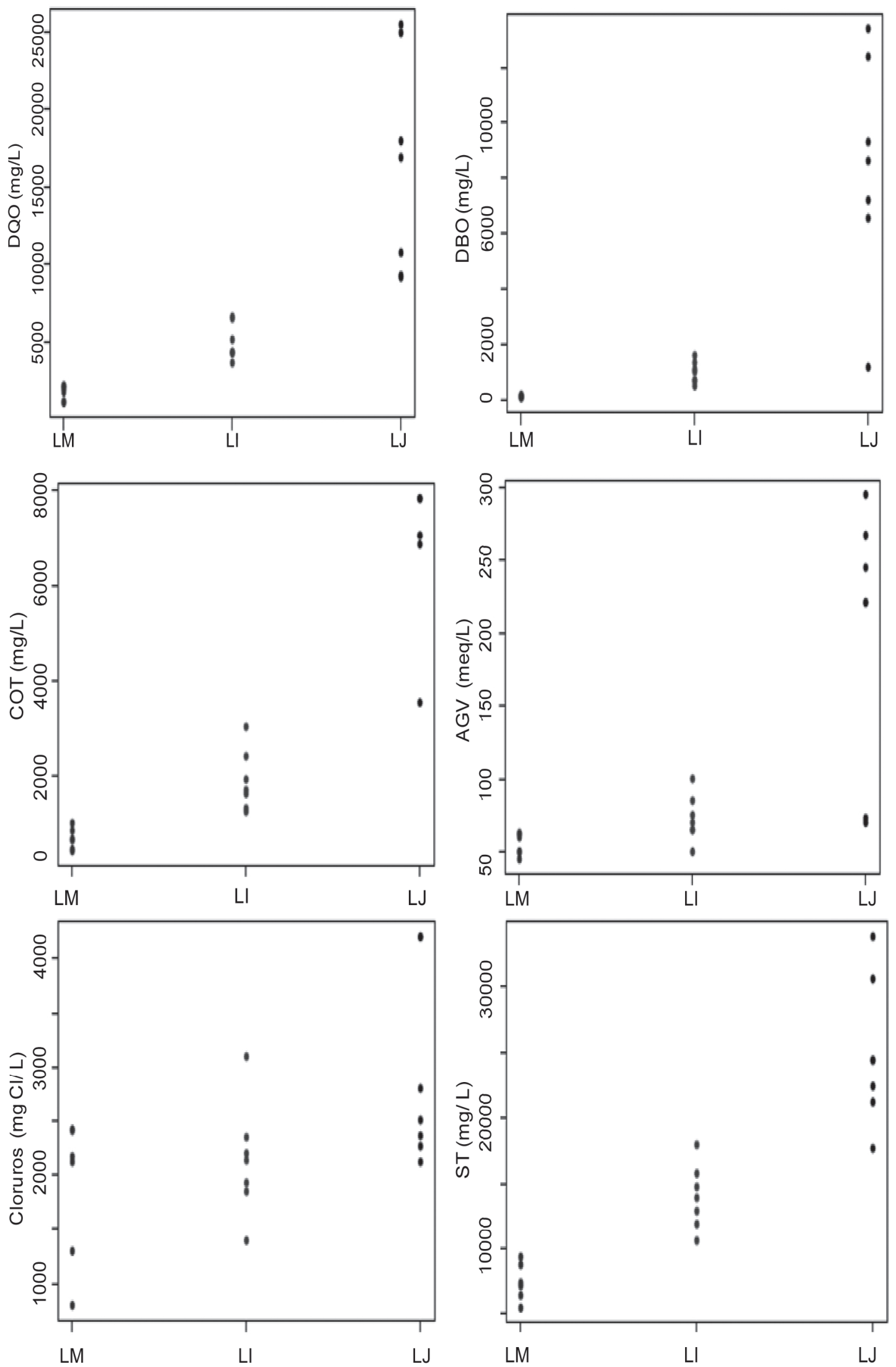

Figura 1. Comportamiento de variables fisicoquímicas. 

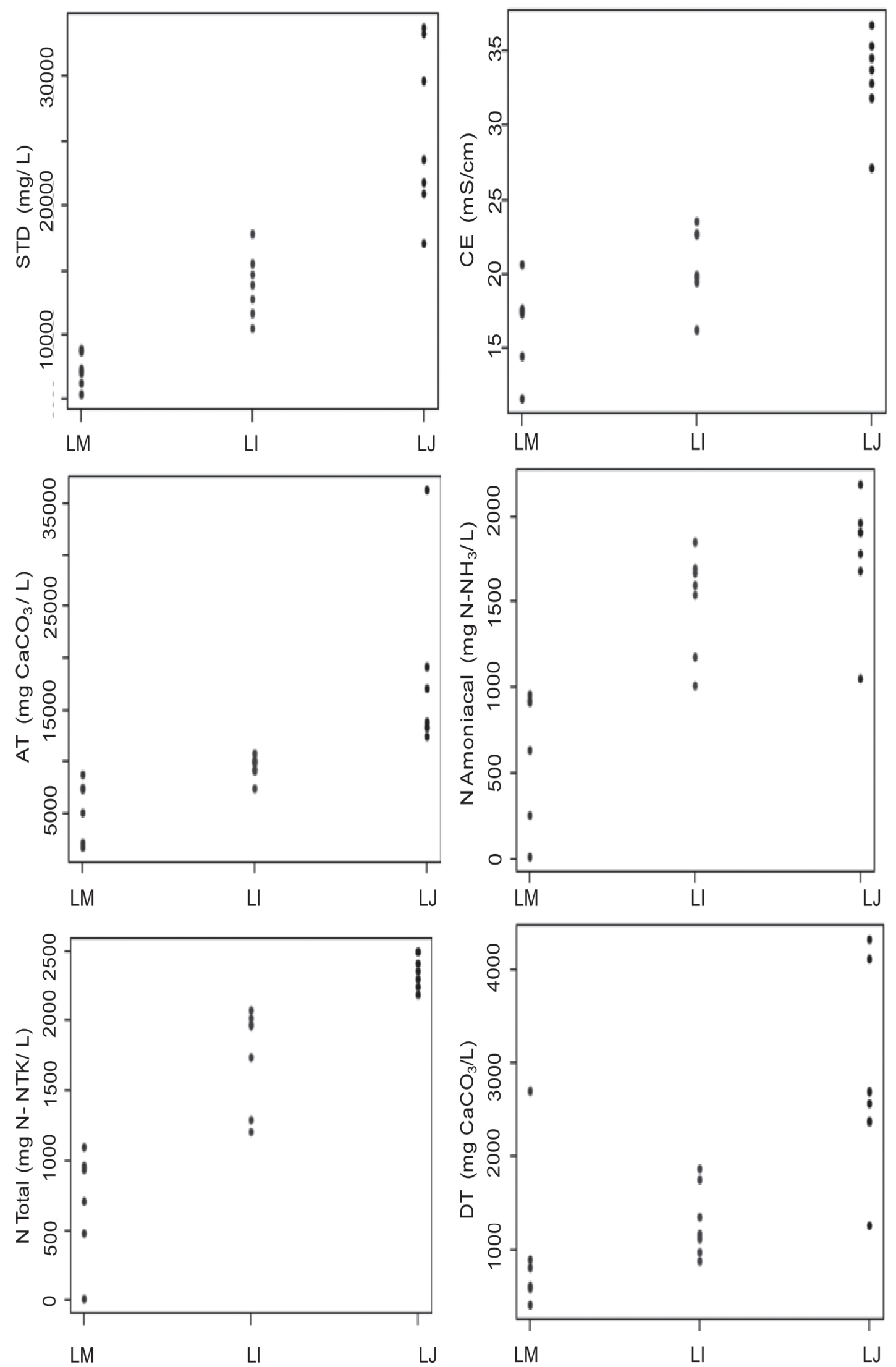

Figura 2. Comportamiento de variables fisicoquímicas. 
En general, las características de los lixiviados estudiados corresponden a lo que diversos autores denominan típicas, de acuerdo con la edad de la celda (LJ: joven con < 5 años; LI: intermedio con un periodo de 5 a 10 años y LM: maduro con periodos mayores a 10 años, respectivamente) (Kang et al. 2002; Kulikowska \& Klimiuk, 2008; Renou et al. 2008; Shouliang et al. 2008).

El pH aumentó con la edad del lixiviado, lo que coincide con la reducción de los AGV, debido a que los rellenos sanitarios jóvenes se encuentran en la fase acidogénica del proceso anaerobio, que conduce a la liberación de grandes cantidades de AGV, que pueden representar hasta el $95 \%$ de la materia orgánica, a diferencia de lo que sucede en rellenos sanitarios maduros, que se encuentran o inician su fase metanogénica, donde los AGV se transforman en metano y en dióxido de carbono (Tchobanoglous et al. 1994; Kurniawan et al. 2006; Renou et al. 2008; Kheradmand et al. 2010).

Parámetros, como la conductividad, la alcalinidad y la dureza, tienen una relación directamente proporcional, ya que dependen de la presencia de iones en los lixiviados (Kang et al. 2002). De esta manera, se observa cómo a medida que aumenta la edad del lixiviado, disminuye la dureza y la alcalinidad. Asimismo, se reduce la presencia de los iones bicarbonato, carbonato, magnesio y calcio, lo cual, disminuye la movilidad iónica y se ve reflejado en valores menores de conductividad.

Con relación a las variables indicadoras de Materia Orgánica (DQO, DBO, y COT), se observó una tendencia a disminuir a medida que aumentaba la edad del lixiviado. La relación DBO/DQO también decreció significativamente, de 0,53 para LJ; 0,16 para LI y 0,09 para LM, lo cual, es indicativo de la disminución de la biodegradabilidad de los lixiviados con respecto de la edad y que coincide con lo encontrado por autores, como Fátima et al. (2012) y Ramírez - Sosa et al. (2013).

En términos del nitrógeno, tanto el total como el amoniacal, disminuyen a medida que aumenta la edad del lixiviado, puesto que en los vertederos jóvenes ocurre la desaminación de los aminoácidos y la destrucción de compuestos orgánicos (Kulikowska \& Klimiuk, 2008). Ziyang et al. (2009) encontraron que la variación del nitrógeno presente en celdas de diferente edad fue alta en los primeros 4 años y se podía estabilizar en los 8 años siguientes; la relación $\mathrm{NH}_{4}{ }^{+}-\mathrm{N} / \mathrm{NT}$ también decreció en el tiempo del 97 al 56\%, indicando que el porcentaje de otras especies, como nitritos y nitratos, se incrementa en los lixiviados maduros.

En los lixiviados estudiados, los valores de amoniaco o amonio no ionizable $\left(\mathrm{NH}_{3}\right)$ presentaron una relación inversa con la edad de la celda, variando entre 187-1090, 237-787 y 41-
$259 \mathrm{mgL}^{-1}$ para LJ, LI y LM, respectivamente (valores promedio 675,470 y $211 \mathrm{mgL}^{-1}$ ). Al igual que el papel de los metales pesados y la alcalinidad, los compuestos nitrogenados presentes en los lixiviados han sido ampliamente estudiados, debido a que se relacionan con la generación de efectos potencialmente inhibitorios o tóxicos (Olivero et al. 2008; Pablos et al. 2011).

En los lixiviados evaluados, los niveles de contaminantes, como HAP's y BTEX estuvieron por debajo de los límites de detección de las técnicas analíticas, debido, probablemente, a que los residuos sólidos depositados en los diferentes rellenos sanitarios son de procedencia urbana, los cuales, normalmente, aportan bajos niveles de estos contaminantes comparados con residuos industriales. Otros factores que influyen en la presencia no cuantificable de HAPs y BTEX son los cambios bioquímicos y procesos físico-químicos, que incluyen la disolución, adsorción, precipitación, dilución, volatilización y otros que influyen en la calidad del lixiviado y que se llevan a cabo en forma natural en el relleno (Kulikowska \& Klimiuk, 2008).

En general, los resultados de los parámetros físico-químicos para los tres lixiviados estudiados presentan importantes relaciones entre la edad de la celda de donde provenían los lixiviados y las variaciones de sus características, encontrándose una relación inversamente proporcional entre la edad de los lixiviados y el potencial de toxicidad, reflejado en la reducción de los niveles de biodegradabilidad, en función de la mayor edad de los lixiviados.

Análisis de Correlaciones: En la tabla 2, se presenta la matriz de correlaciones, en la cual, se pueden observar correlaciones altas entre las variables DBO, DQO, COT y AGV, que era de esperarse, debido a que todas ellas están asociadas a materia orgánica. Adicionalmente, el parámetro que presentó una fuerte relación con un mayor número de variables (DBO, DQO, COT y ST) fue SDT. Por otro lado, la CE presentó un alto grado de correlación con COT, ST y SDT; esta última correlación coincide con la presentada por autores, como Mor et al. (2006), quienes encontraron que la CE y los SDT se encontraban estrechamente relacionados $(0,994)$. Se resalta que el $\mathrm{pH}$ presentó una correlación negativa y débil con todas las variables.

\section{Análisis de Componentes Principales - ACP}

Selección de los componentes principales: En la tabla 3, se presentan los valores propios y de inercia para la selección de los componentes principales. En esta tabla, se observa que con los dos primeros componentes se explica el $80 \%$ de la variabilidad total de las variables bajo estudio y teniendo en cuenta que uno de los criterios para seleccionar el número de componentes es que éstos cubran un porcentaje de 
Tabla 2. Matriz de Correlación de variables fisicoquímicas.

\begin{tabular}{|c|c|c|c|c|c|c|c|c|c|c|c|c|c|}
\hline & $\mathrm{pH}$ & DBO & DQO & COT & ST & SDT & $\mathrm{CE}$ & AT & AGV & N Am & N To & D & $\mathrm{Cl}^{-}$ \\
\hline $\mathrm{pH}$ & 1,00 & & & & & & & & & & & & \\
\hline DBO & $-0,35$ & 1,00 & & & & & & & & & & & \\
\hline DQO & $-0,41$ & $\underline{0,96}$ & 1,00 & & & & & & & & & & \\
\hline COT & $-0,37$ & $\underline{0,94}$ & $\underline{0,89}$ & 1,00 & & & & & & & & & \\
\hline ST & $-0,52$ & $\underline{0,88}$ & $\underline{0,91}$ & $\underline{0,88}$ & 1,00 & & & & & & & & \\
\hline SDT & $-0,51$ & $\underline{0,87}$ & $\underline{0,90}$ & $\underline{0,87}$ & $\underline{0,97}$ & 1,00 & & & & & & & \\
\hline CE & $-0,44$ & 0,78 & 0,83 & $\underline{0,86}$ & $\underline{0,88}$ & $\underline{0,88}$ & 1,00 & & & & & & \\
\hline AT & $-0,33$ & 0,77 & 0,83 & 0,74 & 0,67 & 0,67 & 0,74 & 1,00 & & & & & \\
\hline AGV & $-0,35$ & $\underline{0,89}$ & $\underline{0,88}$ & $\underline{0,88}$ & 0,80 & 0,81 & 0,76 & 0,63 & 1,00 & & & & \\
\hline N Am & $-0,47$ & 0,48 & 0,58 & 0,57 & 0,74 & 0,73 & 0,74 & 0,58 & 0,36 & 1,00 & & & \\
\hline N To & $-0,46$ & 0,65 & 0,71 & 0,76 & 0,82 & 0,81 & $\underline{0,85}$ & 0,65 & 0,62 & 0,93 & 1,00 & & \\
\hline D & $-0,32$ & 0,77 & 0,68 & 0,78 & 0,71 & 0,69 & 0,61 & 0,47 & 0,63 & 0,53 & 0,64 & 1,00 & \\
\hline $\mathrm{Cl}^{-}$ & $-0,06$ & 0,47 & 0,51 & 0,54 & 0,59 & 0,67 & 0,65 & 0,41 & 0,48 & 0,55 & 0,58 & 0,22 & 1,00 \\
\hline
\end{tabular}

D: Dureza; N To: Nitrógeno Total; N Am: Nitrógeno Amoniacal; AT: Alcalinidad Total; CE: Conductividad Eléctrica; SDT: Sólidos Disueltos Totales; ST: Sólidos Totales.

Tabla 3. Valores Propios y coordenadas asociadas a las variables en cada componente.

\begin{tabular}{|l|c|c|c|c|c|c|}
\hline \multirow{2}{*}{ Variables } & \multicolumn{3}{|c|}{ Valores Propios y de Inercia } & \multicolumn{2}{c|}{ Coordenadas asociadas en cada } \\
\cline { 2 - 7 } & Valores propios & Acum & Variabilidad & Componente 1 & Componente 2 & Componente 3 \\
\hline pH & 9,2 & 9,2 & 0,71 & 0,49 & 0,33 & 0,69 \\
\hline DBO & 1,12 & 10,32 & 0,79 & $-0,92$ & 0,36 & $-0,01$ \\
\hline DQO & 0,98 & 11,3 & 0,87 & $-0,94$ & 0,23 & $-0,01$ \\
\hline COT & 0,59 & 11,89 & 0,91 & $-0,94$ & 0,22 & 0,02 \\
\hline ST & 0,51 & 12,4 & 0,95 & $-0,96$ & $-0,04$ & $-0,04$ \\
\hline SDT & 0,21 & 12,61 & 0,97 & $-0,96$ & $-0,06$ & 0,02 \\
\hline CE & 0,16 & 12,77 & 0,98 & $-0,93$ & $-0,12$ & 0,1 \\
\hline AT & 0,12 & 12,89 & 0,99 & $-0,79$ & 0,08 & 0,04 \\
\hline AGV & 0,06 & 12,95 & 1,00 & $-0,85$ & 0,38 & 0,02 \\
\hline N Am & 0,03 & 12,98 & 1,00 & $-0,75$ & $-0,6$ & $-0,01$ \\
\hline N To & 0,01 & 12,99 & 1,00 & $-0,87$ & $-0,38$ & 0,01 \\
\hline D & 0,01 & 13,00 & 1,00 & $-0,75$ & 0,21 & $-0,24$ \\
\hline Cl & 0 & 13,00 & 1,00 & $-0,62$ & $-0,27$ & 0,65 \\
\hline
\end{tabular}


varianza, entre el 80 y 90\% (Díaz, 2007), para efectos de este estudio se eligieron dos componentes.

Correlación de los parámetros con los componentes: En la tabla 3, también se presentan las coordenadas asociadas a las variables para cada una de los componentes. Se resalta que la mayoría de las variables que muestran una alta correlación con el primer componente, están asociadas a materia orgánica (DBO, DQO, COT), además de los ST, SDT y CE; el $\mathrm{pH}$ y los cloruros están altamente correlacionados con el componente tres. Pablos et al. (2011) encontraron en la evaluación de 21 muestras de lixiviados de diferentes rellenos sanitarios, que los tres primeros componentes permitían explicar el $72,55 \%$ de la variabilidad total, siendo cloruros, alcalinidad, conductividad, amoníaco y demanda química de oxígeno, los parámetros que mayor representación tenían en el primer componente.

Correlación entre individuos y variables: En la figura 3, se presenta gráficamente la correlación entre los individuos y las variables con los planos factoriales $1-2$, donde el eje $\mathrm{X}$ es el primer componente y el eje $\mathrm{Y}$, el segundo. Se observa que la mayoría de las mediciones realizadas al lixiviado LJ están relacionadas con los ST y los SDT y con la DBO, DQO y el COT; además, muestra alta relación con el primer componente. Las variables fisicoquímicas Nitrógeno total y amoniacal están relacionadas con el segundo componente principal.

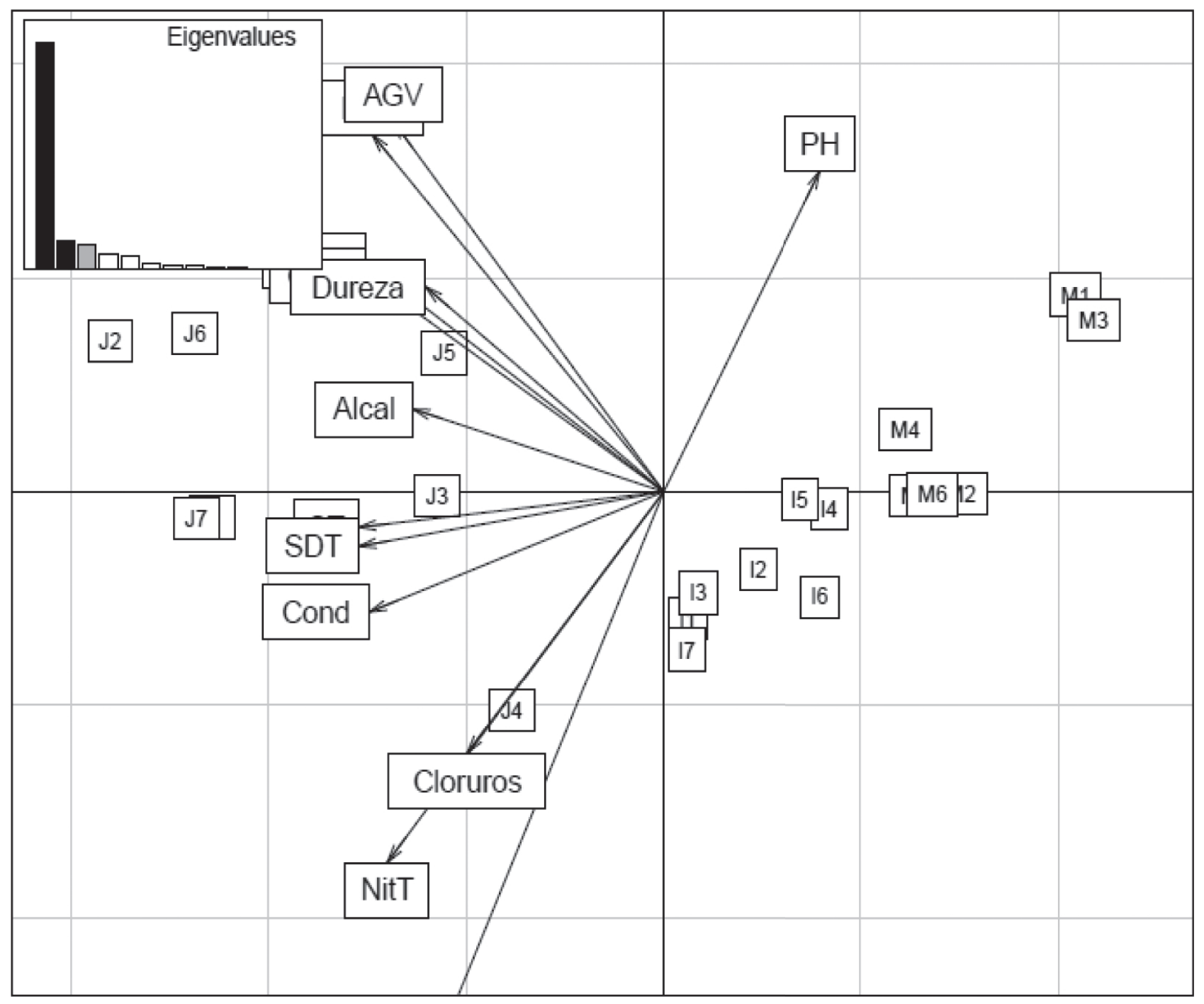

Figura 3. Correlaciones entre individuos y variables en planos $1-2$. 
También es de notar que todas las observaciones tomadas de los lixiviados jóvenes están relacionadas con variables fisicoquímicas, indicando la alta concentración que existe en estos lixiviados, contrario a lo que se puede observar con los lixiviados intermedio y maduro, pues por la edad de éstos presentan bajas concentraciones de las variables fisicoquímicas en cuestión, por lo que es recomendable considerar un mayor número de variables, para tener una caracterización más precisa de estos lixiviados.

Para futuros estudios es recomendable realizar un análisis individual en cada relleno sanitario y con un mayor número de muestras, ya que se pudo observar que el tiempo de funcionamiento de éstos influye en la concentración de las variables fisicoquímicas en sus respectivos lixiviados. La realización de estudios de toxicidad es una herramienta que podría complementar la toma de decisiones para la gestión adecuada de los lixiviados, generados en cada tipo de relleno sanitario.

Se concluye que los resultados de las variables fisicoquímicas medidas a los lixiviados evaluados, muestran que la composición de los lixiviados depende del tiempo de funcionamiento de los rellenos sanitarios y, particularmente de la edad de la celda, en los cuales se originan. En general, se observaron mayores concentraciones de los parámetros fisicoquímicos en los lixiviados más jóvenes, que disminuyeron proporcionalmente con la edad.

En el lixiviado joven, la mayoría de mediciones en el lixiviado están asociadas con las variables fisicoquímicas DBO, DQO, ST, SDT y los AGV's, por lo que este tipo de lixiviados se puede caracterizar mediante estas variables. En lixiviados de mayor edad, las concentraciones medidas disminuyen y la relación entre las variables no es tan fuerte, siendo necesario caracterizar un mayor número de variables, como las indicadoras de material inorgánico y contaminantes específicos, que deberán ser definidos de acuerdo con las características y procedencia de los residuos sólidos depositados en los rellenos sanitarios.

Agradecimientos: Los autores agradecen a la Universidad del Valle por la financiación del Proyecto "Evaluación de la influencia de la edad de rellenos sanitarios municipales sobre la composición y toxicidad de los lixiviados". Conflictos de intereses: El manuscrito fue preparado y revisado con la participación de todos los autores, quienes declaramos que no existe ningún conflicto de intereses que ponga en riesgo la validez de los resultados presentados.

\section{BIBLIOGRAFÍA}

1. ABU-QDAIS, H.A.; HAMODA, M.F.; NEWHAM, J. 1997. Analysis of residential solid waste at generation sites. Waste Manag. Res. 5:395-405.
2. ANTHONISEN, A.C.; LOEHR, R.C.; PRAKASAM, T.B.S.; SRINATH, E.G. 1976. Inhibition of Nitrification by Ammonia and Nitrous Acid. J. Water Pollut Con F. 48:835-852.

3. APHA. 2005. Standard methods for the examination of water and wastewater. $21^{\text {st }}$ ed. Editors APHA, Am. Water Works Assoc. and Water Environment Federation.(Washington, DC). 1368p.

4. AZIZ, S.Q.; AZIZ, H.A.; YUSOFF, M.S.; BASHIR, M.J.K.; UMAR, M. 2010. Leachate characterization in semiaerobic and anaerobic sanitary landfills: A comparative study. J. Environ. Manag. 91:2608-2614.

5. BARTONE, C.R.; BERNSTEIN, J.D. 1993. Improving municipal solid waste management in third world countries. Resourc., Conserv. Recyc. 8:43-54.

6. BOUMECHHOUR, F.; RABAH, K.; LAMINE, C.; SAID, B.M. 2013. Treatment of landfill leachate using Fenton process and coagulation/flocculation. Water Environ. J. 27:114-119.

7. CHESSEL, D.; DUFOUR, A.B.; THIOULOUSE, J. 2004: The ad4 package-I-One-table methods. R. News. 4:5-10.

8. CHRISTENSEN, T.H.; KJELDSEN, P.; ALBRECHTSEN, H.J.; HERON, G.; NIELSEN, P.H.; BJERG, P.L.; HOLM, P.E. 1994. Attenuation of landfill leached pollutants in acquifers. Crit. Rev. Env. Sci. Tec. 24:119202.

9. DÍAZ, L. 2007. Estadística Multivariada: Inferencia y Métodos.Ed. Universidad Nacional. Bogotá D.C. 529p.

10. DRAY, S.; DUFOUR, A.B. 2007. The ade4 package: implementing the duality diagram for ecologists. J. Stat. Softw. 22:1-20.

11. DRAY, S.; DUFOUR, A.B.; CHESSEL, D. 2007. The ad4 package-II: Two-table and k-table methods. R. News. 7:47-52.

12. EL-FADEL, M.; BOU-ZEID, E.; CHAHINE, W.; ALAYLI, B. 2002. Temporal variation of leachate quality from pre-sorted and baled municipal solid waste with high organic and moisture content. Waste Manag. 22:269-282.

13. FÁTIMA, S.; RAFIQ, S.K.; HAI, A. 2012. Effect of depth and age on leachate characteristics of Achan Landfill, Srinagar, Jammu and Kashmir, India. J. Environ. Sci. Tox. Food Technol. 2:4-11. 
14. HOORNWEG, D.; BHADA-TATA, P. 2012. What a waste: A global review of solid waste management. En: Bank, W. (ed.) Urban Development Series Knowledge papers. Washington D.C.: Urban Development and Local Government Unit \& World Bank. 22p.

15. JOHNSON, R.A.; WICHERN, D.W. 2007. Applied Multivariate Statistical Analysis. New Jersey: Prentice Hall. p.457-458.

16. KANG, K.; SHIN, H.S.; PARK, H. 2002. Characterization of humic substances present in landfill leachates with different landfill ages and its implications. Water Res. 36:4023-4032.

17. KARAK, T.; BHAGAT, R.M.; BHATTACHARYYA, P. 2013. Municipal Solid Waste Generation, Composition, and Management: The World Scenario. Crit. Rev. Env. Sci. Tec. 43:215-215.

18. KHERADMAND, S.; KARIMI-JASHNI, A.; SARTAJ, M. 2010.Treatment of municipal landfill leachate using a combined anaerobic digester and activated sludge system. Waste Manag. 30:1025-1031.

19. KJELDSEN, P.; BARLAZ, M.A.; ROOKER, A.P.; BAUN, A.; LEDIN, A.; CHRISTENSEN, T.H. 2002. Present and long-term composition of MSW landfill leachate: a review. Crit. Rev. Env. Sci. Tec. 32:297-336.

20. KULIKOWSKA, D.; KLIMIUK, E. 2008. The effect of landfill age on municipal leachate composition. Biores. Technol. 99:5981-5985.

21. KURNIAWAN, T.A.; LO, W.H.; CHAN, G. 2006. Physico-chemical treatments for removal of recalcitrant contaminants from landfill leachate. J. Hazard Mat. 129:80-100.

22. MOR, S.; RAVINDRA, K.; DAHIYA, R.P.; CHANDRA, A. 2006. Leachate characterization and assessment of groundwater pollution near municipal solid waste landfill site. Environ. Monit. Assess. 118:435-456.

23. OLIVERO, J.; PADILLA, C.; LA ROSA, O.D. 2008. Relationships between physicochemical parameters and the toxicity of leachates from a municipal solid waste landfill. Ecotox. Environ. Safe. 70:294-299.

24. PABLOS, M.V.; MARTINI, F.; FERNÁNDEZ, C.; BABÍN, M.M.; HERRAEZ, I.; MIRANDA, J.; MARTÍNEZ, J.; CARBONELL, G.; SAN-SEGUNDO, L.; GARCÍAHORTIGÜELA, P.; TARAZONA, J.V. 2011. Correlation between physicochemical and ecotoxicological approaches to estimate landfill leachates toxicity. Waste Manag. 31:1841-1847.

25. $R$ CORE TEAM. 2012. R: A language and environment for statistical computing. R Foundation for Statistical Computing, Vienna, Austria.ISBN 3-900051-7-0. URL Disponible desde Internet en: http://www.R-project.org/ (con acceso29/11/2012).

26. RACO, B.; DOTSIKA, E.; BATTAGLINI, R.; BULLERI, E.; DOVERI, M.; PAPAKOSTANTINOU, K. 2013. A Quick and Reliable Method to Detect and Quantify Contamination from MSW Landfills: a Case Study. Water Air Soil Poll. 224:1-18.

27. RAGLE, N.; KISSEL, J.; ONGERTH, J.E.; DEWALLE, F.B. 1995. Composition and Variability of Leachate from Recent and Aged Areas within a Municipal Landfill. Water Environ. Res. 67:238-243.

28. RAMÍREZ-SOSA, D.R.; CASTILLO-BORGES, E.R.; MÉNDEZ-NOVELO, R.I.; SAURI-RIANCHO, M.R.; BARCELÓ-QUINTAL, M.; MARRUFO-GÓMEZ, J.M. 2013. Determination of organic compounds in landfill leachates treated by Fenton-Adsorption. Waste Manag. 33:390-395.

29. RENOU, S.; GIVAUDAN, J.G.; POULAIN, S.; DIRASSOUYAN, F.; MOULIN, P. 2008. Landfill leachate treatment: Review and opportunity. J. Hazard Mat.150:468-493.

30. SHEKDAR, A.V. 2009. Sustainable solid waste management: An integrated approach for Asian countries. Waste Manag. 29:1438-1448.

31. SHOULIANG, H.; BEIDOU, X.; HAICHAN, Y.; LIANSHENG, H.; SHILEI, F.; HONGLIANG, L. 2008. Characteristics of dissolved organic matter (DOM) in leachate with different landfill ages. J. Environ. Sci. 20:492-498.

32. SSPD - SUPERINTENDENCIA DE SERVICIOS PÚBLICOS DOMICILIARIOS. 2010. Situación de la disposición final de los residuos sólidos en Colombia. Diagnóstico 2009. Bogotá D.C.: La Superintendencia. 65p.

33. TCHOBANOGLOUS, G.; THEISEN, H.; VIGIL, S. 1994. Gestión integral de residuos sólidos. Ciudad de México. McGraw-Hill/ Interamericana de España S.A. p.471.

34. THEMELIS, N.J. 2003. An overview of the global wasteto-energy industry. Waste Manag. World. 4:40-47. 
35. THIOULOUSE, J.; DRAY, S. 2009. ad4TkGUI: ad4 Tcl/Tk Graphical User Interface. R package version 0.2-5. Disponible desde internet en http://CRAN.R-project. org/package $=$ ade4TkGUI (con acceso 3/12/2012).

36. WISZNIOWSKI, J.; ROBERT, D.; SURMACZ-GORSKA, J.; MIKSCH, K.; WEBER, J.V. 2006. Landfill leachate treatment methods: A review. Environ. Chem. Lett. 4:51-61.
37. ZIYANG, L.; YOUCAI, Z.; TAO, Y.; YU, S.; HUILI, C.; NANWEN, Z. 2009. Natural attenuation and characterization of contaminants composition in landfill leachate under different disposing ages. Sci. Total Environ. 407:3385-3391.

Recibido: Marzo 12 de 2013

Aceptado: Enero 27 de 2014

\section{Como citar:}

Torres-Lozada, P.; Barba-Ho, L.E.; Ojeda, C.; Martínez, J.; Castaño, J. 2014. Influencia de la edad de lixiviados sobre su composición físico-química y su potencial de toxicidad. Rev. U.D.C.A Act. \& Div. Cient. 17(1): 245-255. 
